\section{Toben statt schniefen}

\section{Die Allergieentwicklung in der Kindheit wird von zahlreichen Faktoren beeinflusst. Eine Kohortenstudie hat jetzt eine Korrelation zwischen körperlicher Betätigung und der Prävalenz der allergischen Rhinitis gezeigt.}

$\mathrm{D}$ ie Daten einer Kohorte von 2.429 sächsischen Kindern, die zu Beginn der Studie in den Jahren 1992 und 1993 fünf bis 14 Jahre alt waren, wurden über maximal zwölf Jahre hinweg verfolgt. Bei der Anfangserhebung machten die Eltern schriftlich unter anderem Angaben zur körperlichen Aktivität ihrer Kinder: 1.923 wurden als aktiv, 364 als mäßig aktiv und 142 als inaktiv eingestuft. Zudem wurden das Vorliegen von spezifischen IgE-Antikörpern und von allergischer Rhinitis überprüft. Über den Untersuchungszeitraum hinweg konnten 1.703 Kinder zumindest noch ein weiteres Mal untersucht werden. Mithilfe von Regressionsmodellen wurde versucht, einem Zusammenhang zwischen körperlicher Aktivität und der
Häufigkeit von Heuschnupfen auf die Spur zu kommen.

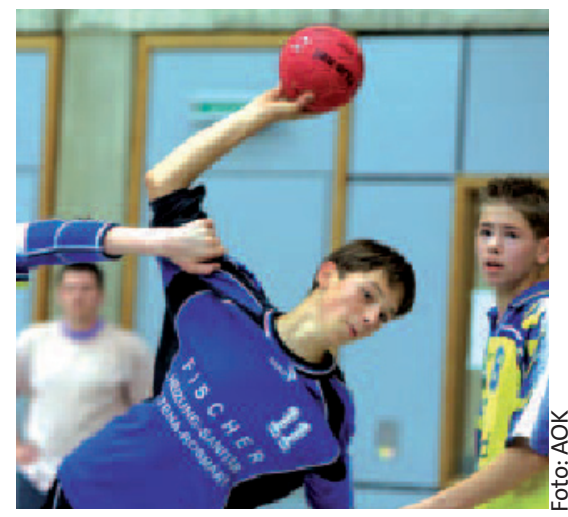

Im Mitgliedsbeitrag für den Sportverein ist möglicherweise auch gleich die Allergieprävention mit enthalten.

\title{
Verhindern Impfungen Allergien?
}

Impfstoffe bewirken nicht nur eine spezifische Reaktion des Immunsystems auf die applizierte Vakzine, sondern auch weitere immunologische Veränderungen. In einer tierexperimentellen Studie untersuchten Forscher der Berliner Charité, welchen Einfluss Impfungen auf eine Ovalbuminsensibilisierung haben.

mmunkompetente Mäuse (BALB/c-

Stamm) erhielten intrakutan entweder Diphtherie- und Tetanustoxoid, eine Dreifachkombination aus Diphtherie- und Tetanustoxoid sowie Bordetella-pertussis-Vakzine oder eine salzhaltige Kontrolllösung appliziert. Anschließend wurden die Versuchstiere intraperitoneal gegen Ovalbumin sensibilisiert. Alle Mäuse mussten sich einer wiederholten inhalativen Allergenprovokation mit Hühnereiweißaerosol unterziehen und wurden danach untersucht.

Das Gesamt-IgE lag bei den geimpften Mäusen signifikant höher als bei den nicht geimpften Tieren. Das
Ovalbumin-spezifische $\operatorname{IgE}$ dagegen war bei den geimpften Mäusen signifikant niedriger. Das spezifische $\mathrm{IgG}_{2 \mathrm{a}}$ lag bei den Mäusen, die gegen Diphtherie und Tetanus geimpft waren, niedriger als bei den Kontrolltieren. Die zusätzliche Applikation der Pertussisvakzine führte dagegen zu einer Erhöhung dieses Wertes. Mononukleäre Zellen von geimpften Mäusen produzierten in vitro signifikant weniger Interleukin- $4,-5$ und -10 sowie mehr Interferon- $\gamma$ als die entsprechenden Zellen nicht geimpfter Mäuse.

Alle Mäuse, die Vakzine erhalten hatten, wiesen in der bronchoalveolären Lavage signifikant weniger Eosinophile
Es zeigte sich, dass körperlich inaktive Kinder bereits zu Beginn der Untersuchung signifikant häufiger unter einer saisonalen allergischen Rhinitis litten als aktive. Diese Assoziation blieb über den gesamten Untersuchungszeitraum erhalten. Insgesamt stieg die Prävalenz der allergischen Rhinitis von 4,3\% zu Beginn der Untersuchung auf 23,1\% am Ende an. Das Vorliegen von allergenspezifischem IgE korrelierte dagegen nicht mit der körperlichen Aktivität der Kinder. Neben der Intensität der körperlichen Betätigung waren eine Allergie der Eltern und das Lebensalter an sich Prädiktoren für eine saisonale allergische Rhinitis.

Fazit: Kinder, die sich viel bewegen, sind genauso häufig sensibilisiert wie körperlich inaktive Kinder. Sie leiden aber signifikant seltener unter einer allergischen Rhinitis.

$f k$

Kohlhammer Y et al. Influence of physical inactivity on the prevalence of hay fever. Allergy 2006; 61: 1310-15 auf. Die Lymphozytenzahl dagegen war interessanterweise in der Gruppe der dreifach geimpften Mäuse am höchsten. Bei der bronchialen Reaktivität war zwischen den nicht geimpften Mäusen und den gegen Diphtherie und Tetanus geimpften Tieren kein Unterschied zu beobachten. Dagegen unterdrückte die Dreifach-Impfkombination komplett die Entwicklung einer bronchialen $\mathrm{Hy}$ perreaktivität.

Fazit: In dieser Studie führten Impfungen mit Diphtherie- und Tetanustoxoid alleine oder in Kombination mit einer Pertussisvakzine bei Mäusen insgesamt zu einer Abschwächung allergischer Reaktionen. Offenbar kann die Vakzinierung eine TH2-Immunantwort abschwächen, wenn Impfung und Sensibilisierung zeitlich nah beieinander liegen.

Grüber C et al. Common vaccine antigens inhibit allergen-induced sensitization and airway hyperresponsiveness in a murine model. Allergy 2006; 61: 820-7 